\title{
Detection of inherited and acquired hemostatic disorders in surgical patients
}

\author{
Marco Ranucci, MD
}

Received: 8 May 2016/Accepted: 16 June 2016/Published online: 24 June 2016

(C) Canadian Anesthesiologists' Society 2016

In this issue of the Journal, Bonhomme et al. ${ }^{1}$ propose a structured questionnaire (HEMSTOP; Hematoma, hEmorrhage, Menorrhagia, Surgery, Tooth extraction, Obstetrics, Parents) to facilitate preoperative identification of surgical patients with hemostatic disorders requiring specific measures to reduce perioperative bleeding. The HEMSTOP is a seven-point questionnaire, with each question requiring a simple yes/no answer to attain a score from 0-7 depending on the number of positive answers. The authors retrospectively assessed the HEMSTOP questionnaire in patients referred to hemostasis specialists. These specialists subsequently recommended whether or not hemostatic precautions were required in these patients prior to surgery. Additionally, the authors prospectively tested the HEMSTOP questionnaire in healthy volunteers. The authors identified a cut-off value of 2 or higher for the HEMSTOP score, which yielded a sensitivity of $89.5 \%$ and a specificity of $98.6 \%$ for the diagnosis of a bleeding disorder. Assuming a prevalence of bleeding disorders of $1 \%$, these values correspond to a negative predictive value of $99.9 \%$ and a positive predictive value of $39.1 \%$. Importantly, the questionnaire was not intended to identify bleeding disorders related to ongoing medications and known pathological conditions associated with increased bleeding risk. Therefore, the clinical utility of the HEMSTOP questionnaire should be considered within the context of preoperative detection of as yet undiagnosed (and therefore likely mild) inherited or

M. Ranucci, MD ( $\square)$

Department of Cardiothoracic and Vascular Anesthesia and ICU, IRCCS Policlinico San Donato, Via Morandi 30, San Donato Milanese, Milan, Italy

e-mail: cardioanestesia@virgilio.it acquired bleeding disorders unrelated to more well-known pathological conditions associated with disrupted hemostasis.

Apart from hemophilia and other inherited bleeding disorders leading to severe bleeding diathesis, coagulation factor deficiencies account for 3-5\% of all inherited coagulation disorders and include deficiencies in fibrinogen and factors (F) II, V, V + VIII, VII, X, XI, and XIII. ${ }^{2,3}$ Their prevalence is very low, ranging from 1:500,000 for FVII deficiency to 1:2,000,000 for prothrombin and FXIII deficiency. ${ }^{2}$ Inherited platelet function disorders are much more common. Indeed, primary platelet function disorders include a vast number of pathological entities affecting adhesion and aggregation receptors such as can be seen in the Bernard-Soulier syndrome and Glanzmann thrombasthenia. The true prevalence of these conditions is still unknown and the bleeding symptoms may vary from mild to severe. ${ }^{4}$ Inherited von Willebrand disease (vWD) is the most common inherited bleeding disorder, and approximately $1 \%$ of the population have defects of the von Willebrand factor (vWF) consistent with the diagnostic criteria for vWD. Nevertheless, only $1 \%$ of these patients will develop a significant bleeding episode during their life span. ${ }^{5}$ It is likely, however, that many people may experience undiagnosed vWD-related bleeding episodes. ${ }^{5}$

In the setting of a surgical population, there are other acquired bleeding disorders that are often underdiagnosed. Aortic valve stenosis (AS), the most common heart valve disease, is associated with a bleeding tendency in about $20 \%$ of these patients. ${ }^{6}$ Gastrointestinal bleeding is the most dangerous acquired bleeding disorder, and the association between AS and gastrointestinal bleeding constitutes Heyde's syndrome, ${ }^{7}$ which is found in about $8 \%$ of AS patients. ${ }^{6,8}$ In 1997, Warkentin et al. reported 
that AS patients may suffer from an acquired type $2 \mathrm{~A}$ von Willebrand syndrome due to a qualitative defect of $\mathrm{vWF}$ associated with the loss of vWF high-molecular-weight multimers. ${ }^{9}$ This defect seems to be related to the increased shear stress produced by the stenotic valve and usually disappears after correction of the stenosis. ${ }^{10}$ Therefore, preoperative identification of this type of acquired $\mathrm{vWD}$ may be important for patients scheduled for AS correction through conventional surgery or transcatheter aortic valve replacement. Similarly, an acquired vWD is reported in other types of pathological heart conditions associated with an elevated shear stress, such as prosthetic mitral valve leak, hypertrophic obstructive cardiomyopathy, and mitral valve regurgitation.

Another acquired bleeding disorder that may be found in surgical patients is acquired hemophilia $(\mathrm{AH})$, a rare disease characterized by the presence of autoantibodies against factor VIII. The most common cause of $\mathrm{AH}$ is malignancy, ${ }^{11,12}$ thus exposing cancer-related surgical patients to an increased risk of bleeding.

All together, the combination of inherited and acquired undiagnosed bleeding disorders likely approximates a prevalence of $1 \%$, as hypothesized by the authors when determining the negative and positive predictive values of a HEMSTOP score of 2 or higher. Therefore, a preoperative effort to identify those patients with an elevated risk for bleeding is reasonable from a clinical perspective, and the authors are to be congratulated for their work. Importantly, routine laboratory tests inadequately identify patients with primary or secondary platelet function disorders, and alternative screening tools are required. Nevertheless, as the authors acknowledge, there are several remaining issues to address before the HEMSTOP questionnaire can be recommended as routine preoperative hemostatic assessment screening in surgical patients.

The first and most important point is related to the clinical application of the HEMSTOP score as a guide to refer patients for hematology consultation. The authors recommend consultation when the HEMSTOP score is 2 or higher. I am unconvinced, however, that there is sufficient evidence to support this recommendation; moreover, I question whether this is practically feasible on a largescale basis. Certainly, it would seem reasonable to employ this strategy for surgical procedures associated with medium-high risk of bleeding.

The high negative predictive value of the HEMSTOP score $<2$ is certainly impressive, but in clinical terms, this score should be judged in terms of its positive rather than its negative predictive value. Indeed, the aim of the questionnaire is not to exclude the existence of a bleeding disorder, but to alert to the possibility of an existing bleeding disorder and thus to refer the patient to a specialist. From this perspective, the authors' reported positive predictive value of $39 \%$ needs to be assessed ideally using a larger series of patients. In this setting, the following question needs to be answered, "How many patients in an unselected surgical population will have a HEMSTOP score of 2 or higher?" In the study by Bonhomme et al., ${ }^{1}$ this was reported in only $1.4 \%$ of healthy volunteers. Nevertheless, this rate may be higher in patients undergoing surgery. Should this be the case, it begs the question whether we can refer such a considerable number of our patients to a hematology specialist for consultation. Until we have a clear idea of the actual HEMSTOP score in a large surgical patient population, the feasibility of this strategy remains uncertain.

Another point of concern is the role of anticoagulants and antiplatelet drugs on the HEMSTOP score. In this present study, ${ }^{1}$ the HEMSTOP questionnaire was not concerned with the bleeding risk associated with the ongoing use of these types of drugs. Nevertheless, in the real world, many patients take various combinations of medications that interfere with coagulation up until a few days before surgery. As the HEMSTOP questionnaire explores the bleeding history, it is possible that the responses are influenced by the effects of anticoagulant medications. Finally, there is the potential for a sex-based bias, since a cut-off value of 2 out of 5 in males cannot be considered equivalent to a value of 2 out of 7 in females.

Given these caveats, it is likely that the HEMSTOP questionnaire provides an easy and practical tool for preoperative screening of bleeding risk in surgical patients. Furthermore, its application to a large series of patients will further clarify its role in the detection of inherited and acquired bleeding disorders.

\section{Le dépistage des troubles de l'hémostase héréditaires ou acquis chez les patients chirurgicaux}

Dans ce numéro du Journal, Bonhomme et coll. ${ }^{1}$ présentent un questionnaire structuré du nom de HEMSTOP (pour «Hematoma, hEmorrhage, Menorrhagia, Surgery, Tooth extraction, Obstetrics, Parents », soit Hématome, hémorragie, ménorragie, chirurgie, extraction dentaire, obstétrique, et parents). L'objectif du questionnaire est de faciliter l'identification préopératoire des patients chirurgicaux souffrant de troubles de l'hémostase et nécessitant la prise de mesures particulières afin de réduire les saignements périopératoires. Le questionnaire HEMSTOP comporte sept points et la réponse à chaque question est soit 
affirmative, soit négative; le score total se calcule en fonction du nombre de réponses positives, et se situe donc entre 0 et 7 . L'évaluation du questionnaire HEMSTOP s'est faite de façon rétrospective auprès de patients référés à des spécialistes de l'hémostase. Ces spécialistes ont par la suite recommandé la prise de précautions hémostatiques ou non - avant la chirurgie des patients ainsi identifiés comme courant un risque. Les auteurs ont également testé le questionnaire HEMSTOP de manière prospective en le soumettant à des volontaires sains. Les auteurs ont identifié une valeur limite $<2$ pour le score HEMSTOP, valeur avec laquelle ils ont obtenu une sensibilité de $89,5 \%$ et une spécificité de $98,6 \%$ pour diagnostiquer un trouble de saignement. Supposant une prévalence de $1 \%$ des troubles de saignement, ces valeurs correspondent à une valeur prédictive négative de $99,9 \%$ et une valeur prédictive positive de $39,1 \%$. Il est important de noter que le questionnaire n'avait pas pour but d'identifier les troubles de saignement liés à la prise constante de médicaments ou à des conditions pathologiques connues associées à un risque accru de saignement. Par conséquent, la pertinence clinique du questionnaire HEMSTOP doit être perçue dans le contexte du dépistage préopératoire de troubles de saignement hérités ou acquis et jusqu'alors non diagnostiqués (donc probablement légers) et sans rapport à des conditions pathologiques mieux connues associées à une hémostase déréglée.

Hormis l'hémophilie et les autres troubles de saignement hérités entraînant une diathèse hémorragique grave, les déficiences en facteurs de coagulation sont à l'origine de 3-5\% de tous les troubles de coagulation hérités et comprennent les déficiences en fibrinogène et en facteurs (F) II, V + VIII, VII, X, XI et XIII. ${ }^{2,3}$ Leur prévalence est extrêmement faible, allant de 1:500 000 pour les déficiences de FVII à 1:2000 000 pour les déficiences de prothrombine et de FXIII. ${ }^{2}$ Les troubles hérités de la fonction plaquettaire sont beaucoup plus répandus. En effets, les troubles primaires de la fonction plaquettaire comprennent un nombre important d'entités pathologiques affectant les récepteurs d'adhésion et d'agrégation comme cela se manifeste en cas de syndrome de Bernard-Soulier et de thrombasthénie de Glanzmann. La véritable prévalence de ces conditions demeure inconnue et les symptômes de saignements vont de légers à graves. ${ }^{4}$ La maladie de von Willebrand $(\mathrm{mvW})$ est le trouble de saignement hérité le plus fréquemment observé, et approximativement $1 \%$ de la population souffre de défauts du facteur de von Willebrand (fvW) répondant aux critères diagnostiques de mvW. Cependant, seulement $1 \%$ de ces patients souffriront d'un épisode hémorragique important durant leur vie. ${ }^{5}$ Il est néanmoins probable que plusieurs personnes souffrent d'épisodes de saignements liés à une mvW non diagnostiquée. ${ }^{5}$
Lorsqu'il s'agit de population chirurgicale, il existe d'autres troubles de saignement acquis souvent non diagnostiqués. La sténose de la valve aortique (SA), la maladie valvulaire cardiaque la plus répandue, est associée à une tendance de saignement chez environ $20 \%$ de ces patients. ${ }^{6}$ Les saignements gastro-intestinaux sont le trouble de saignement acquis le plus dangereux, et le syndrome de Heyde, une association entre SA et saignements gastro-intestinaux, ${ }^{7}$ est un syndrome que l'on retrouve chez environ $8 \%$ des patients atteints de SA. ${ }^{6,8}$ En 1997, Warkentin et coll. rapportaient que les patients atteints de SA pouvaient souffrir d'un syndrome de von Willebrand acquis de type 2 en raison d'un défaut qualitatif $\mathrm{du} \mathrm{fvW}$ associé à la perte de multimères du fvW à haut poids moléculaire. ${ }^{9}$ Ce défaut semble être lié à l'augmentation de la force de cisaillement causée par la valve sténosée et disparaît généralement après correction de la sténose. ${ }^{10}$ Ainsi, l'identification préopératoire de ce type de mvW acquise pourrait s'avérer fort utile chez les patients devant subir une correction de la SA par chirurgie conventionnelle ou un remplacement de la valve aortique via un cathéter. De la même façon, une mvW acquise a été rapportée dans d'autres types de cardiopathies associées à une force de cisaillement élevée, comme en cas de fuite d'une valve mitrale prothétique, de cardiomyopathie obstructive hypertrophique et de régurgitation de la valve mitrale.

Un autre trouble de saignement acquis que l'on peut observer chez les patients chirurgicaux est l'hémophilie acquise (HA), une maladie rare caractérisée par la présence d'anticorps contre le facteur VIII. Les tumeurs malignes sont la cause la plus répandue de $\mathrm{HA},{ }^{11,12}$ exposant ainsi les patients de chirurgie oncologique à un risque accru de saignement.

Pris ensemble, la combinaison des troubles de saignements non diagnostiqués, qu'ils soient hérités ou acquis, avoisine probablement une prévalence de $1 \%$, comme les auteurs le suggèrent lorsqu'ils déterminent les valeurs prédictives négatives et positives d'un score HEMSTOP de 2 ou plus. Il est par conséquent raisonnable, d'un point de vue clinique, de déployer des efforts en période préopératoire afin d'identifier les patients courant un risque élevé de saignement, et les auteurs doivent être félicités pour leurs travaux. Il est important de souligner que les tests de laboratoire de routine ne sont pas optimaux pour identifier les patients atteints de troubles de la fonction plaquettaire primaires ou secondaires, et d'autres outils de dépistage sont nécessaires. Toutefois, comme les auteurs le précisent, plusieurs questions doivent encore être soulevées et trouver réponse avant que le questionnaire HEMSTOP ne puisse être recommandé comme outil de dépistage préopératoire de l'hémostase auprès des patients chirurgicaux.

Le point essentiel est lié à l'application clinique du score HEMSTOP en tant que guide pour référer des patients en hématologie. Les auteurs recommandent une consultation 
lorsque le score HEMSTOP est supérieur ou égal à 2. Je demeure peu convaincu, toutefois, qu'il existe suffisamment de données probantes pour appuyer une telle recommandation; de plus, je me demande si cela est faisable d'un point de vue pratique sur une vaste échelle. Il semble indubitablement raisonnable d'avoir recours à cette stratégie lorsqu'on a affaire à des interventions chirurgicales associées à un risque modéré à élevé de saignement.

La valeur prédictive négative élevée du score HEMSTOP $<2$ est certes impressionnante; toutefois, d'un point de vue clinique, ce score devrait être jugé selon sa valeur prédictive positive plutôt que négative. En effet, l'objectif de ce questionnaire n'est pas d'exclure l'existence d'un trouble de saignement, mais bien de mettre en lumière la possibilité d'un trouble de saignement existant, et donc de référer un patient à un spécialiste. Vue sous cet angle, la valeur prédictive positive rapportée par les auteurs de $39 \%$ devrait idéalement être évaluée auprès d'une plus grande série de patients. Dans ce contexte, il faut trouver une réponse à la question suivante : «Combien de patients, dans une population chirurgicale non triée, auront un score HEMSTOP supérieur ou égal à 2? » Dans l'étude de Bonhomme et coll., ${ }^{1}$ un tel score n'a été rapporté que chez $1,4 \%$ des volontaires sains. Ce taux pourrait toutefois être plus élevé dans une population chirurgicale. Si tel était le cas, la question de savoir si l'on peut référer un tel nombre de patients à un spécialiste en hématologie pour une consultation se pose. Tant que nous n'aurons pas une idée précise du score HEMSTOP véritable observé dans une importante population de patients chirurgicaux, la faisabilité de cette stratégie demeurera incertaine.

Une autre inquiétude touche à l'impact potentiel des anticoagulants et médicaments antiplaquettaires sur le score HEMSTOP. Dans l'étude présentée ici, ${ }^{1}$ le questionnaire HEMSTOP ne s'est pas intéressé au risque de saignement associé à l'utilisation ininterrompue de ce type de médicaments. Dans le monde réel toutefois, un nombre considérable de patients prennent différents cocktails médicamenteux qui interfèrent avec la coagulation jusqu'à quelques jours avant leur chirurgie. Étant donné que le questionnaire HEMSTOP explore les antécédents de saignement, il est possible que les réponses de ces patients soient influencées par les effets de médicaments anticoagulants. Enfin, il est possible qu'il y ait un biais fondé sur le sexe : il est évident qu'une valeur limite de 2 sur 5 chez les hommes ne peut être considérée équivalente à une valeur de 2 sur 7 chez les femmes.

Étant donné ces réserves, il est probable que le questionnaire HEMSTOP constituera un outil facile et pratique pour le dépistage préopératoire d'un risque de saignement chez des patients chirurgicaux. En outre, son application à une importante série de patients permettra de mieux clarifier son rôle dans le dépistage des troubles de saignement hérités ou acquis.

Conflicts of interest None declared.

Editorial responsibility This submission was handled by Dr. Steven Backman, Associate Editor, Canadian Journal of Anesthesia.

\section{Conflit d'intérêt Aucun.}

Responsabilité éditoriale Cet article a été traité par Dr Steven Backman, rédacteur adjoint, Journal canadien d'anesthésie.

\section{References}

1. Bonhomme F, Boehlen F, Clergue $F$, de Moerloose $P$. Preoperative hemostatic assessment: a new and simple bleeding questionnaire. Can J Anesth 2016; 63: this issue. doi:10.1007/ s12630-016-0688-9

2. Peyvandi F, Palla R, Menegatti M, Mannucci PM. Introduction: rare bleeding disorders: general aspects of clinical features, diagnosis, and management. Semin Thromb Hemost 2009; 35: 349-55.

3. James $P$, Salomon $O$, Mikovic D, Peyvandi F. Rare bleeding disorders - bleeding assessment tools, laboratory aspects and phenotype and therapy of FXI deficiency. Haemophilia 2014; 20(Suppl 4): 71-5.

4. Israels SJ, El-Ekiaby M, Quiroga T, Mezzano D. Inherited disorders of platelet function and challenges to diagnosis of mucocutaneous bleeding. Haemophilia 2010; 16(Suppl 5): 152-9.

5. De Jong A, Eikenboom J. Developments in the diagnostic procedures for von Willebrand disease. J Thromb Haemost 2016; 14: 449-60.

6. Vincentelli A, Susen $S$, Le Tourneau T, et al. Acquired von Willebrand syndrome in aortic stenosis. N Engl J Med 2003; 349: 343-9.

7. Heyde E. Gastrointestinal bleeding in aortic stenosis. N Engl $\mathbf{J}$ Med 1958; 259: 196.

8. Natorska J, Mazur P, Undas A. Increased bleeding risk in patients with aortic valvular stenosis: from new mechanisms to new therapies. Thromb Res 2016; 139: 85-9.

9. Warkentin TE, Moore JC, Morgan DG. Aortic stenosis and bleeding gastrointestinal angiodysplasia: is acquired von Willebrand's disease the link? Lancet 1992; 340: 35-7.

10. Solomon C, Budde U, Schneppenheim S, et al. Acquired type 2A von Willebrand syndrome caused by aortic valve disease corrects during valve surgery. Br J Anaesth 2011; 106: 494-500.

11. Borg JY, Guillet B, Le Cam-Duchez V, Goudemand J, Levesque H, SACHA Study Group. Outcome of acquired haemophilia in France: the prospective SACHA (Surveillance des Auto antiCorps au cours de l'Hémophilie Acquise) registry. Haemophilia 2013; 19: 564-70.

12. Tiede A, Klamroth R, Scharf RE, et al. Prognostic factors for remission of and survival in acquired hemophilia A (AHA): results from the GTH-AH 01/2010 study. Blood 2015; 125: 1091-7. 